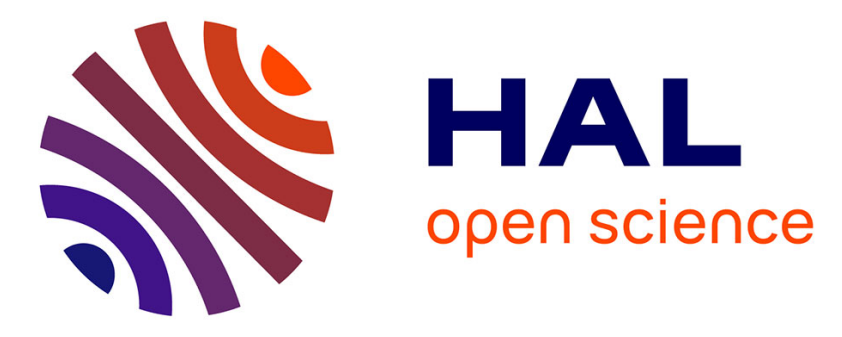

\title{
Parameter-tolerant design of high contrast gratings
} Christyves Chevallier, Nicolas Fressengeas, Joel Jacquet, Guilhem Almuneau, Youness Laaroussi, Olivier Gauthier-Lafaye, Laurent Cerutti, Frédéric Genty

\section{To cite this version:}

Christyves Chevallier, Nicolas Fressengeas, Joel Jacquet, Guilhem Almuneau, Youness Laaroussi, et al.. Parameter-tolerant design of high contrast gratings. Photonics West, SPIE, Feb 2015, San Francisco, United States. pp.93720N-93720N-8, 10.1117/12.2081595 . hal-01132683

\section{HAL Id: hal-01132683 \\ https://hal.science/hal-01132683}

Submitted on 16 Apr 2020

HAL is a multi-disciplinary open access archive for the deposit and dissemination of scientific research documents, whether they are published or not. The documents may come from teaching and research institutions in France or abroad, or from public or private research centers.
L'archive ouverte pluridisciplinaire HAL, est destinée au dépôt et à la diffusion de documents scientifiques de niveau recherche, publiés ou non, émanant des établissements d'enseignement et de recherche français ou étrangers, des laboratoires publics ou privés. 


\title{
Parameter-tolerant design of high contrast gratings
}

\author{
Christyves Chevallier $^{a, b}$, Nicolas Fressengeas ${ }^{c}$, Joel Jacquet ${ }^{d}$, Guilhem Almuneau $^{e}$, Youness \\ Laaroussi $^{e}$, Olivier Gauthier-Lafaye ${ }^{e}$, Laurent Cerutti ${ }^{e}$ and Frédéric Genty ${ }^{a}$ \\ ${ }^{a}$ Supélec, Laboratoire Matériaux Optiques, Photonique et Systèmes EA 4423, \\ 2 rue Edouard Belin, Metz, F-57070, France \\ ${ }^{b}$ Georgia Tech - CNRS, International Joint Research Lab, UMI 2958, \\ 2-3 rue Marconi, Metz, F-57070, France \\ ${ }^{c}$ Université de Lorraine, Laboratoire Matériaux Optiques, Photonique et Systèmes EA 4423, \\ 2 rue Edouard Belin, Metz, F-57070, France \\ ${ }^{d}$ Captoor, F-57070 Chieulles, France \\ ${ }^{e}$ Laboratoire d'Analyse et d'Architecture des Systèmes, \\ Centre National de la Recherche Scientifique, \\ 7 Avenue du Colonel Roche, F-31077 Toulouse, France

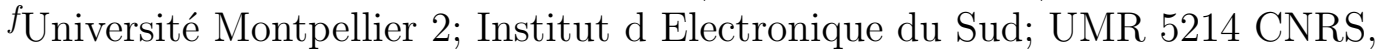 \\ 860, rue Saint Priest, F-34095 Montpellier Cedex 5, France
}

\begin{abstract}
This work is devoted to the design of high contrast grating mirrors taking into account the technological constraints and tolerance of fabrication. First, a global optimization algorithm has been combined to a numerical analysis of grating structures (RCWA) to automatically design HCG mirrors. Then, the tolerances of the grating dimensions have been precisely studied to develop a robust optimization algorithm with which high contrast gratings, exhibiting not only a high efficiency but also large tolerance values, could be designed. Finally, several structures integrating previously designed HCGs has been simulated to validate and illustrate the interest of such gratings.
\end{abstract}

Keywords: High contrast grating, Near wavelength diffraction grating, Grating mirror, Robust optimization, Tolerance analysis

\section{INTRODUCTION}

High contrast gratings (HCG) are usually 1D photonic structures made of two materials with a large index step $(\sim 2)$. When the periodicity is in the range of the wavelength, diffraction is possible only into the $0^{\text {th }}$ order and only a few Bloch modes are propagative and can interact with each others. In the high contrast grating scenario, the incoming lightwave is normal to the slab and the mode propagation is studied in a perpendicular direction to the slab plane. ${ }^{1,2}$ The coupling between the propagating modes and the outside waves as well as the resonances of the modes between the two slab interfaces can be tailored by adjusting the grating dimensions and the index contrast to give a wide range of optical properties. ${ }^{3}$ High contrast gratings have thus become a powerful tool for many application such as high-Q resonators ${ }^{4}$ and highly efficient sensors, planar lenses ${ }^{5}$ and beam steering cite, MEMS ${ }^{6,7}$ broadband terahertz absorber with large angle tolerance. ${ }^{8}$ But the most powerful application of HCG is their ability to produce high reflectivity and large bandwidth mirrors ${ }^{9-12}$ which is for instance a promising solution for full color reflective display for e-book readers. ${ }^{13}$ HCG mirrors have originally been developed for VCSEL devices : with only two subwavelength layers, they can surpass Bragg reflectors performances in term of high reflectivity. Moreover, due to their 1D symmetry, HCG can exhibit a high polarization selectivity which is an important advantage to prevent polarization instabilities in the VCSEL emitted beam.

Further author information: (Send correspondence to Christyves Chevallier) Christyves Chevallier: E-mail: cchevallier@georgiatech-metz.fr, Telephone: +33(0)387203945 


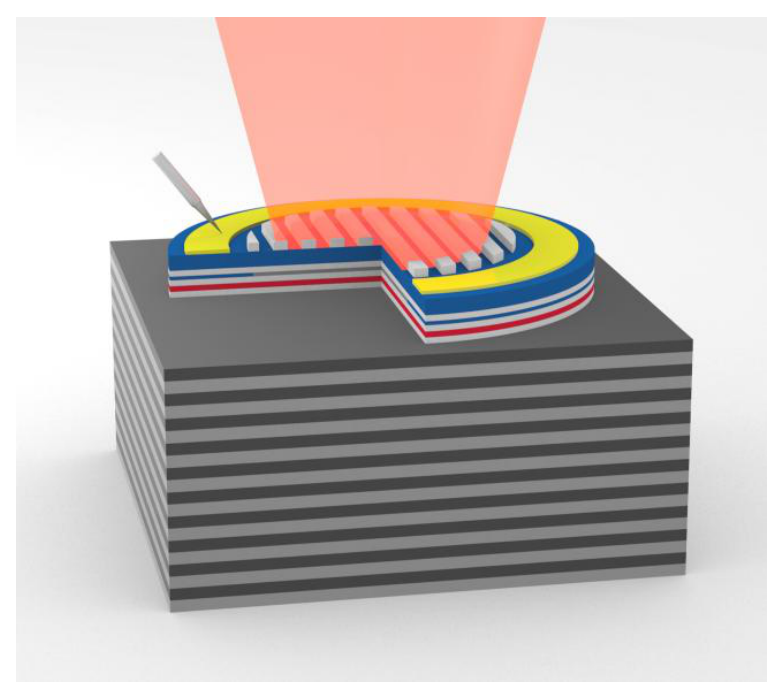

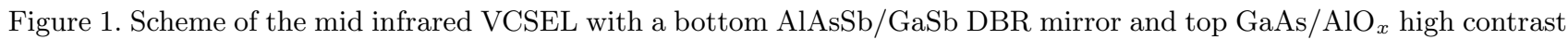
grating mirror.

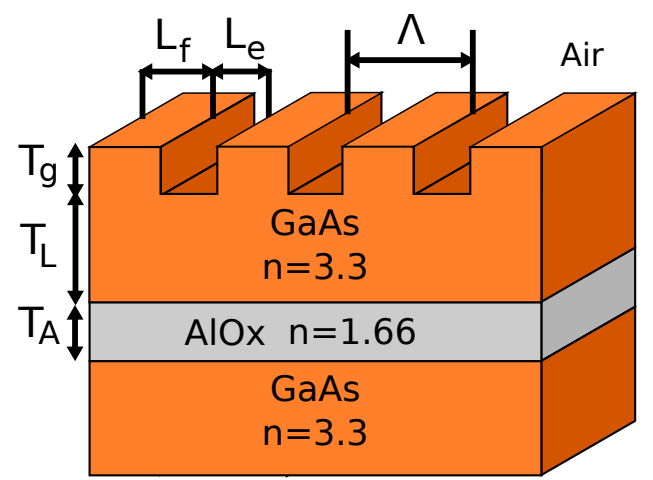

Figure 2. Scheme of the $\mathrm{GaAs} / \mathrm{AlO}_{x}$ mirror. The grating dimensions are adjusted by an optimization algorithm to exhibit reflectivity higher than $99.5 \%$ for a VCSEL application at $2.3 \mathrm{\mu m}$.

In this work, a HCG is designed to enhance VCSEL performances in the mid-infrared wavelength range. For wavelength above $2 \mu \mathrm{m}$, VCSELs based on the antimony alloy are a very attractive light source to develop and enhance numerous applications such as polluting gas sensing. However, due to the large thickness of the device with mirrors as thick as $9 \mu \mathrm{m}$, antimony alloy VCSEL emission is still limited at $2.6 \mu \mathrm{m} .{ }^{14,15}$ High contrast gratings are thus a promising new type of mirror to reduce the structure thickness, introduce polarization stability and develop devices emitting at larger wavelengths. The design of the HCG is done here by taking into account VCSEL mirror requirements through the use of an optimization algorithm. Since high contrast grating mirrors have sub-micrometric dimensions with square-shaped patterns and the VCSELs require high quality mirrors, the fabrication has to be accurately controlled in the nanometric range. ${ }^{16}$ The tolerance with respect to the errors of fabrication is an important aspect and has to be taken into account during the design of the structure to achieve a robust and efficient mirror. ${ }^{17-19}$

In this paper, we present the design of a GaAs HCG combined with an $\mathrm{AlO}_{x}$ sublayer as low index material to replace the top DBR of mid infrared GaSb-based VCSEL. In a first part, an optimization algorithm is used to find the best dimensions of the HCG structure. Then, the tolerance of the geometrical parameters of the optimum design with respect to the errors of fabrication are numerically investigated. In a second part, an anti-optimization algorithm is combined to the optimization process to develop a robust optimization algorithm. High contrast gratings are thus optimized to exhibit not only high efficiency but also large tolerance values. 
Table 1. Boundaries of the search space and tolerance requirements for the robust optimization algorithm.

\begin{tabular}{llll}
\hline Parameter & Minimum value & Maximum value & Minimum tolerance \\
\hline$T_{g}$ & $500 \mathrm{~nm}$ & $1100 \mathrm{~nm}$ & $\Delta T_{g}> \pm 20 \mathrm{~nm}$ \\
$F F$ & 0.35 & 0.55 & $\Delta F F> \pm 0.02$ \\
$T_{A}$ & $300 \mathrm{~nm}$ & $400 \mathrm{~nm}$ & $\Delta T_{A}> \pm 50 \mathrm{~nm}$ \\
$\Lambda$ & $900 \mathrm{~nm}$ & $1300 \mathrm{~nm}$ & $\Delta \Lambda> \pm 3 \mathrm{~nm}$ \\
$T_{L}$ & $50 \mathrm{~nm}$ & $1000 \mathrm{~nm}$ & $\Delta T_{L}> \pm 1 \mathrm{~nm}$
\end{tabular}

\section{HCG-VCSEL FABRICATION}

For a mid infrared emission between 2 and $3 \mu \mathrm{m}$, a VCSEL structure is grown by molecular beam epitaxy. The device is composed of five GaInAsSb quantum wells in a cavity composed by a Bragg mirror made from 24 pairs of $\mathrm{AlAsSb} / \mathrm{GaSb}^{20}$ and high contrast grating on top (Figure 1). To enhance the device performances, an electro-optic confinement solution has been introduce with the use of an oxide aperture. Since oxidation of the antimonide alloy (AlSb) has not returned encouraging results for its application as a confinement layer, a metamorphic approach with oxidation of AlGaAs layer is used. ${ }^{21}$ A GaAs layer is thus grown on top of the Sb-based cavity of the VCSEL and the HCG can be obtained thanks to the high contrast between AlOx low index material $(n \sim 1.7)$ with low absorption ${ }^{22}$ and high index of GaAs $(n \sim 3.3)$. This technological approach presents the advantage of using mature industrial process such as reactive ion etching (RIE) for the grating fabrication and wet oxidation for the AlOx layer. In this configuration, the GaAs grating layer is chosen to be not completely etched to prevent delamination during the oxidation process and enhance mirror performances ${ }^{16}$ as shown on Figure 2. The HCG presented here is thus made from a GaAs grating of thickness $T_{g}$ with a fill factor $F F$ on top of a GaAs sublayer of thickness $T_{L}$ and a low index AlOx layer of thickness $T_{A}$.

\section{VCSEL MIRROR REQUIREMENTS}

Compared to other semiconductor lasers, VCSEL devices have a very thin gain region and require high $Q$ cavities made from high reflectivity mirrors higher than $99.5 \%$ with typical bandwidth of $150 \mathrm{~nm}$ in the mid infrared. Besides the mirror effect, a polarization selectivity is wanted to prevent polarization instabilities of the emitted beam which is an well known problem in VCSEL structures due to their circular symmetry. The high reflectivity of $99.5 \%$ is chosen for the transverse magnetic (TM) polarization which is perpendicular to the grating slabs. The transverse electric polarization (TE) is kept lower than $90 \%$ for the whole high reflectivity bandwidth to prevent any lasing for this polarization mode.

\section{TECHNOLOGICAL CONSTRAINTS}

The fabrication of the HCG structure is constrained by technological considerations. The aluminum oxide layer thickness $T_{A}$ is limited between 300 and $400 \mathrm{~nm}$ around the optimum ${ }^{23}(2 k-1) \lambda / 4$ to prevent optical losses and delamination during the wet oxidation process. The fill factor $F F$ of the grating is limited to values between $35 \%$ and $55 \%$ by the e-beam lithography process and the RIE process used to ensure a well defined square shape pattern of the grating slabs.

Besides boundaries on the HCG dimensions, tolerances on the structure parameters have to be taken into account to ensure that the design is feasible and keep high performances despite the pitfalls of the manufacturing process. The etching of the grating slab is the most critical step in the process flow since it is done without the use of a stop layer. The tolerances on the parameter $T_{g}$ and $F F$ are thus wanted to be larger than $\Delta T_{g}>20 \mathrm{~nm}$ and $\Delta F F>2 \%$. The AlOx layer thickness is also a parameter which requires large tolerance values since the thickness changes during the oxidation process and is not easily predictable. This tolerance is set to $\Delta T_{A}>50 \mathrm{~nm}$ as it can be seen in Table 1 which summarized all the parameters limitations. The other dimensions $T_{L}$ or $\Lambda$ have been given empirical values but could be linked to technological requirements as well. 


\section{ROBUST OPTIMIZATION ALGORITHM}

The HCG design can be done with a theoretical analysis of the Bloch mode resonances inside the grating, ${ }^{2}$ but we chose here to solve an inverse problem by computing the reflectivity of the mirror with a numerical analysis and adjust the grating parameters to increase its performances. This method allows to design easily more complex structures which are not ideal HCG gratings and include the previously defined VCSEL requirements during the design process.

\subsection{The optimization algorithm}

An optimization algorithm has been used to automate the search of the most efficient mirror design in a same way that it can be done manually with a study of the evolution of the reflectivity of the mirror with respect to its dimensions. ${ }^{24}$ To automate the search, optimization algorithms require to describe the problem with the use of a mathematical function. A figure of merit $M F$ has thus been defined to represent quantitatively the mirror quality as a VCSEL application point of view :

$$
M F=\frac{\Delta \lambda}{\lambda_{0}} \frac{1}{N} \sum_{\lambda=\lambda_{1}}^{\lambda_{2}} R_{T M}(\lambda) g(\lambda)
$$

The $M F$ function mainly represents the normalized high reflectivity bandwidth of the mirror, defined as the wavelength range $\Delta \lambda=\lambda_{2}-\lambda_{1}$ around $\lambda_{0}$ where the reflectivity is larger than $99.5 \%$ for TM mode and below $90 \%$ for the TE mode. The normalized bandwidth is multiplied by a Gaussian weighted average of the transverse magnetic reflection coefficients $R_{T M}$ of the bandwidth to ensure the centering around $\lambda_{0}$. Reflection spectra of the mirror are computed by rigorous coupled wave analysis (RCWA) ${ }^{25}$ for transverse magnetic and transverse electric polarizations.

Several optimization algorithm are available to search for the maximum of a function and the choice of the best algorithm is done with respect to the function characteristics. In our case, the $M F$ function has many local maximas and the use of Newton like optimization algorithm is not possible. The class of global optimization algorithm is well suited to solve such problems. For instance, simulated annealing, ${ }^{26}$ genetic algorithm ${ }^{27}$ and particle swarm ${ }^{19,28}$ have already shown their ability to design HCG efficiently. In this work, a particle swarm optimization (PSO) algorithm have been chosen due to its ease of use and implementation. PSO algorithm is based on a set of particles which are potential candidates sharing their knowledge of optimum positions when exploring the search space. In our case, the search space is defined by the set of design parameters $\Omega=\left\{\Lambda, T_{g}\right.$, $\left.F F, T_{A}, T_{L}\right\}$ and one point of this search space defines one design solution. The particles of the swarm move inside the search space at each iteration with a velocity $v_{i, p}$ :

$$
v_{i, p}=v_{i-1, p}+c_{l} *\left(x_{p}^{l}-x_{i-1, p}\right)+c_{g} *\left(x_{p}^{g}-x_{i-1, p}\right)
$$

The velocity $v_{i, p}$ at the iteration $i$ of the particle $p$ of the swarm is composed of 3 terms. Firstly, the inertia of the particle is taken into account by keeping the velocity of the previous iteration $v_{i-1, p}$. Secondly, a local velocity term moves the particle toward the local best position $x_{p}^{l}$ known by the particle $p$. Finally, the swarm concept is created by sharing the best position of all particles thanks to the global best position $x_{p}^{g}$ which creates

a global velocity term. The parameters $c_{l}$ and $c_{g}$ are two weights for the local and global velocities which are randomly chosen from a uniform law in the range $[0,2]$ at each particle move. ${ }^{29}$

\subsection{The anti-optimization algorithm}

Even if the optimization algorithm succeed in finding efficient HCG mirrors, a detailed study of the optimum found by the algorithm can show that this point is not realistic from a fabrication point of view due to its very low tolerance. ${ }^{30}$ The robustness can be enhanced manually after the optimization but this task becomes difficult when done simultaneously on several parameters and does not ensure to combine the most efficient and robust design solution. A robust solution corresponds to a design which keeps high performances for large fabrication errors, in other terms, which keeps a high value of its figure of merit $M F$ in the vicinity of the point in the search 
space $\Omega$. To ensure that the algorithm results in the most efficient and robust solution, optimization methods can be written to include tolerance during the search process. One of them, the anti-optimization, ${ }^{31}$ looks for the performance of each solution under the worst case scenario of fabrication. The ideal case is to look for the minimum value in the vicinity with a minimization algorithm such as Newton like method. The vicinity of the point is defined in our case as the space $\Delta X=\left\{\Delta T_{g}, \Delta F F, \Delta T_{A}, \Delta \Lambda, \Delta T_{L}\right\}$ which represents the estimation of the errors that can be made during the fabrication process. Thus, the minimum value of the $M F$ function in the space $X \pm \Delta X$ corresponds to the worst case scenario of fabrication.

An original algorithm has been written to combine the PSO algorithm with an anti optimization method. A new figure of merit $M F^{\prime}$ has been defined to take into account the tolerance :

$$
M F^{\prime}(X)=\frac{1}{2}\left(\min _{\Delta X} M F(X \pm \Delta X)+M F(X)\right)(1+\eta)
$$

$$
\forall(X \pm \Delta X) \in \Omega
$$

The $M F^{\prime}$ function is mainly the average of the performances of the current design $M F(X)$ and the performances of the worst case scenario of fabrication for this design $M F(X \pm \Delta X)$ given the user-defined tolerance specifications $\Delta X$. The search for the worst case scenario is done by the the search of the minimum of the figure of merit $M F$ in the subspace $X \pm \Delta X$. The use of an optimization algorithm to search for that minimum must be done for each evaluation of the function $M F^{\prime}$ and would be time consuming since the evaluation of the figure of merit $M F$ of one $X$ value takes up to 30 seconds.

The anti-optimization algorithm has been designed to be as efficient as possible by decreasing the number of $M F$ function evaluations. First, a memoization method has been introduced to store in memory the results of the $M F(X)$ function for each point $X$ met during the optimization. Thus, if the $M F$ function is evaluated several times, the computing will be done only the first one. The probability to evaluate twice the same design $X$ during an optimization is low, however, this method allows us to simplify the anti-optimization process. Instead of using a minimization algorithm to search for the worst case scenario, the search for the minimum is done by looking into the memorized solutions if a point is in the vicinity $\pm \Delta X$ of the point $X$ being evaluated. To ensure that the vicinity of the point $X$ is explored enough, a routine is executed which evaluates the extreme values $X \pm \Delta X$ of the best solution found by the robust optimization algorithm.

By evaluating the robustness of only the best solutions during the optimization, the PSO algorithm has to compare solutions which have been tested in tolerance and solutions which have not yet been. Since the evaluation in tolerance is done by looking for the worst case scenario with the search of the minimum of $M F(X \pm \Delta X)$ and results in the average between the $M F$ function of the optimum and the worst case, a parameter $\eta$ has been added in the $M F^{\prime}$ definition. This parameter corresponds to a percentage of the process of tolerance evaluation. Thus, efficient mirrors which keep good performances within user-defined variation ranges will have a larger figure of merit $M F^{\prime}$ than non tolerant structures. A competition between optimization and anti-optimization is thus used to enhance the efficiency of the robust algorithm by decreasing the number of points evaluated and the computational cost.

\section{RESULTING DESIGNS}

The execution of the robust optimization algorithm has been done to adjust the parameters of the GaAs/AlOx grating structure defined previously. The boundary of the search space and tolerance requirements on the structure dimensions are summarized in Table 1 and come either from technological limitations as described previously for AlOx thickness $T_{A}$ and fill factor $F F$ or chosen arbitrarily from empirical rules. ${ }^{24}$

The resulting mirror which dimensions are given in Table 2 exhibits a very high reflectivity and polarization selective bandwidth of $425 \mathrm{~nm}$ (Figure 3). The tolerance evaluation of the parameters shows a good robustness with an optimum value well centered within large variation ranges. The grating thickness $T_{g}$ and Fill Factor $F F$ tolerances are very large with $T_{g}=668 \pm 70 \mathrm{~nm}$ and $F F=0.5351 \pm 0.0630$. A statistic study with 30000 tests of the tolerance of the optimum by varying simultaneously all the design parameters with $\Delta T_{g}= \pm 20 \mathrm{~nm}$, $\Delta F F= \pm 0.02, \Delta T_{A}= \pm 50 \mathrm{~nm}, \Delta \Lambda= \pm 3 \mathrm{~nm}$ and $\Delta T_{L}= \pm 1 \mathrm{~nm}$ have returned 0 mirror with less than $99.5 \%$ of TM reflectivity at $\lambda_{0}=2300 \mathrm{~nm}$. 
Table 2. Optimum and tolerance values obtained by the robust optimization algorithm.

\begin{tabular}{llll}
\hline & Optimum & Tolerances for $R_{T M}>99.5 \%$ at $\lambda=2.3 \mu \mathrm{m}$ \\
\hline$T_{g}$ & $668 \mathrm{~nm}$ & $598 \mathrm{~nm}<T_{g}<738 \mathrm{~nm}$ & $\Delta T_{g}= \pm 70 \mathrm{~nm}$ \\
$F F$ & 0.5351 & $0.4361<F F<0.5981$ & $\Delta F F= \pm 0.063$ \\
$T_{A}$ & $360 \mathrm{~nm}$ & $228 \mathrm{~nm}<T_{A}$ & $\Delta T_{A}= \pm 132 \mathrm{~nm}$ \\
$\Lambda$ & $1098 \mathrm{~nm}$ & $993 \mathrm{~nm}<\Lambda<1162 \mathrm{~nm}$ & $\Delta \Lambda= \pm 64 \mathrm{~nm}$ \\
$T_{L}$ & $282 \mathrm{~nm}$ & $240 \mathrm{~nm}<T_{L}<331 \mathrm{~nm}$ & $\Delta T_{L}= \pm 42 \mathrm{~nm}$ \\
\hline$\lambda_{0}$ & $2290 \mathrm{~nm}$ & & \\
$\Delta \lambda$ & $425 \mathrm{~nm}$ & & \\
$\Delta \lambda / \lambda_{0}$ & $18.6 \%$ & &
\end{tabular}

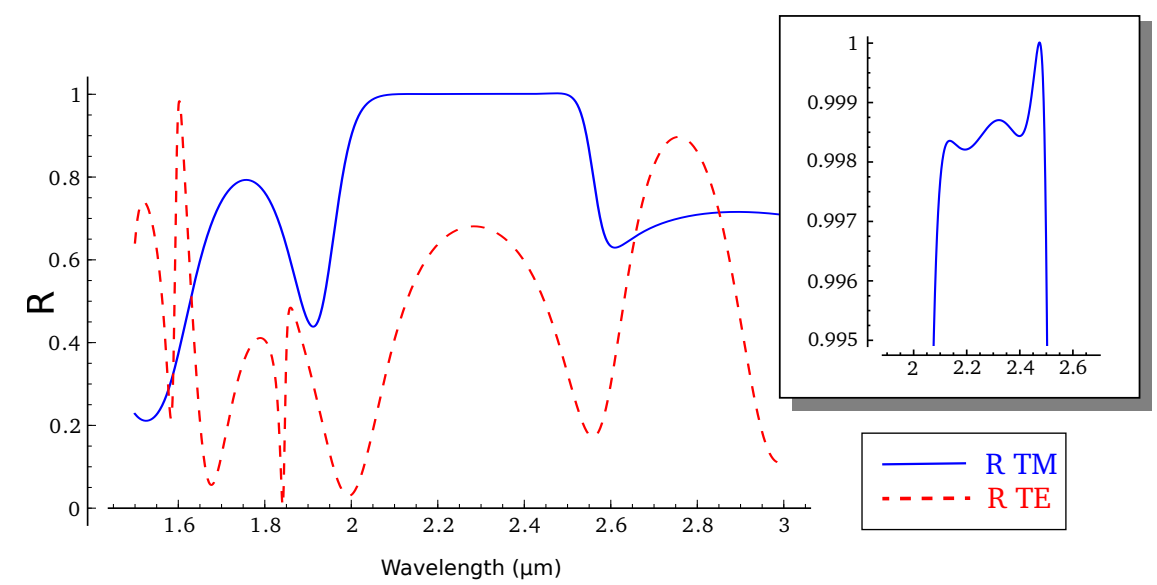

Figure 3. Reflection spectra of the robust HCG mirror. The inset exhibits a large $99.5 \%$ high reflectivity bandwidth of $425 \mathrm{~nm}$ centered at $2290 \mathrm{~nm}$ for the TM coefficient (blue) with a good polarization selectivity by keeping $R_{T E}<70 \%$ (dashed red).

\section{CONCLUSION}

An original robust optimization algorithm for high contrast grating design has been presented in this work which searches not only for the best solution but takes also into account the accuracy of the manufacturing technology. The algorithm has shown its ability to design HCG mirrors for a VCSEL application at $2.3 \mu \mathrm{m}$ while taking into account user-defined technological constraints. The execution of the robust optimization algorithm returns not only efficient mirrors with $99.5 \%$ high reflectivity for $425 \mathrm{~nm}$ large bandwidth but also fulfills the robustness requirements with large tolerance values of more than $10 \%$ on the most critical parameters. This design method can be quickly adapted to design other high contrast grating structures for other manufacturing conditions by only adapting the figure of merit and the technological constraints.

\section{ACKNOWLEDGMENTS}

The authors thank the French ANR for financial support in the framework of Marsupilami project (ANR09-BLAN-0166-03) and IES and LAAS (France), partners of LMOPS/Supélec in this project. This work was also partly funded by the InterCell grant (http://intercell.metz.supelec.fr) by INRIA and Région Lorraine (CPER2007).

\section{REFERENCES}

[1] Lalanne, P., Hugonin, J. P., and Chavel, P., "Optical properties of deep lamellar gratings: A coupled bloch-mode insight," J. Lightwave Technol. 24, 2442 (Jun 2006).

[2] Karagodsky, V., Sedgwick, F. G., and Chang-Hasnain, C. J., "Theoretical analysis of subwavelength high contrast grating reflectors," Opt. Express 18, 16973-16988 (Aug 2010).

[3] Chang-Hasnain, C. J. and Yang, W., "High-contrast gratings for integrated optoelectronics," Adv. Opt. Photon. 4, 379-440 (Sep 2012). 
[4] Zhou, Y., Moewe, M., Kern, J., Huang, M. C., and Chang-Hasnain, C. J., "Surface-normal emission of a high-Q resonator using a subwavelength high-contrast grating," Opt. Express 16, 17282-17287 (Oct 2008).

[5] Fattal, D., Li, J., Peng, Z., Fiorentino, M., and Beausoleil, R. G., "Flat dielectric grating reflectors with focusing abilities," Nat Photon 4, 466-470 (07 2010).

[6] Hane, K., Kobayashi, T., Hu, F.-R., and Kanamori, Y., "Variable optical reflectance of a self-supported Si grating," Applied Physics Letters 88(14), 141109 (2006).

[7] Huang, M. C. Y., Zhou, Y., and Chang-Hasnain, C. J., "A nanoelectromechanical tunable laser," Nat. Photon. 2(3), 180-184 (2008).

[8] Jindal, S. and Kumar, M., "Broadband and polarization insensitive design of terahertz absorber with highindex contrast grating on soi chip," Optical and Quantum Electronics , 1-10 (2014).

[9] Goeman, S., Boons, S., Dhoedt, B., Vandeputte, K., Caekebeke, K., Van Daele, P., and Baets, R., "First demonstration of highly reflective and highly polarization selective diffraction gratings (GIRO-gratings) for long-wavelength VCSELs," Photonics Technology Letters, IEEE 10, 1205 -1207 (sept. 1998).

[10] Mateus, C., Huang, M., Chen, L., Chang-Hasnain, C., and Suzuki, Y., "Broad-band mirror (1.12-1.62 $\mu \mathrm{m})$ using a subwavelength grating," Photonics Technology Letters, IEEE 16, 1676 -1678 (july 2004).

[11] Wu, T. T., Syu, Y. C., Wu, S. H., Chen, W. T., Lu, T. C., Wang, S. C., Chiang, H. P., and Tsai, D. P., "Sub-wavelength gan-based membrane high contrast grating reflectors," Opt. Express 20, 20551-20557 (Aug 2012).

[12] Foley, J. M., Itsuno, A. M., Das, T., Velicu, S., and Phillips, J. D., "Broadband long-wavelength infrared $\mathrm{Si} / \mathrm{SiO}_{2}$ subwavelength grating reflector," Opt. Lett. 37, 1523-1525 (May 2012).

[13] Liu, V. and Fan, S., "S4: A free electromagnetic solver for layered periodic structures," Computer Physics Communications 183(10), 2233 - 2244 (2012).

[14] Ducanchez, A., Cerutti, L., Grech, P., and Genty, F., "GaSb-based monolithic EP-VCSEL emitting above 2.5 m.," Electron. Lett. 44(23), 1357-1359 (2008).

[15] Arafin, S., Bachmann, A., Kashani-Shirazi, K., and Amann, M.-C., "Electrically pumped continuous-wave vertical-cavity surface-emitting lasers at $2.6 \mu \mathrm{m}, "$ Applied Physics Letters 95(13), 131120 (2009).

[16] Chevallier, C., Fressengeas, N., Genty, F., and Jacquet, J., "Optimized sub-wavelength grating mirror design for mid-infrared wavelength range," Appl. Phys. A-Mater. 103(4), 1139-1144 (2011).

[17] Chevallier, C., Fressengeas, N., Genty, F., and Jacquet, J., "Optimized $\mathrm{Si} / \mathrm{SiO}_{2}$ high contrast grating mirror design for mid-infrared wavelength range: Robustness enhancement," Optics ÉLaser Technology 44(3), 626 $-630(2012)$.

[18] Zhou, Y., Huang, M., and Chang-Hasnain, C., "Large fabrication tolerance for VCSELs using high-contrast grating," Photonics Technology Letters, IEEE 20, $434-436$ (march15, 2008).

[19] Wu, H., Mo, W., Hou, J., Gao, D., Hao, R., Jiang, H., Guo, R., Wu, W., and Zhou, Z., "A high performance polarization independent reflector based on a multilayered configuration grating structure," Journal of Optics 12(4), 045703 (2010).

[20] Laaroussi, Y., Chevallier, C., Genty, F., Fressengeas, N., Cerutti, L., Taliercio, T., Gauthier-Lafaye, O., Calmon, P.-F., Reig, B., Jacquet, J., and Almuneau, G., "Oxide confinement and high contrast grating mirrors for mid-infrared vcsels," Opt. Mater. Express 3, 1576-1585 (Oct 2013).

[21] Laaroussi, Y., Almuneau, G., Sanchez, D., and Cerutti, L., "Efficient lateral confinement by an oxide aperture in a mid-infrared GaSb-based vertical light-emitting source," Journal of Physics D: Applied Physics 44(14), 142001 (2011).

[22] Ravaro, M., Guillotel, E., Dû, M. L., Manquest, C., Marcadet, X., Ducci, S., Berger, V., and Leo, G., "Nonlinear measurement of mid-infrared absorption in alo[sub x] waveguides," Applied Physics Letters 92(15), 151111 (2008).

[23] Almuneau, G., Condé, M., Gauthier-Lafaye, O., Bardinal, V., and Fontaine, C., "High reflectivity monolithic sub-wavelength diffraction grating with GaAs/AlOx stack," Journal of Optics 13(1), 015505 (2011).

[24] Mateus, C., Huang, M., Deng, Y., Neureuther, A., and Chang-Hasnain, C., "Ultrabroadband mirror using low-index cladded subwavelength grating," IEEE Photon. Technol. Lett. 16(2), 518-520 (2004).

[25] Bienstman, P. et al., "CAMFR." http://camfr.sourceforge.net/ (2007). 
[26] Bisaillon, E., Tan, D., Faraji, B., Kirk, A., Chrowstowski, L., and Plant, D. V., "High reflectivity air-bridge subwavelength grating reflector and Fabry-Perot cavity in AlGaAs/GaAs," Opt. Express 14, 2573-2582 (Apr 2006).

[27] Shokooh-Saremi, M. and Magnusson, R., "Particle swarm optimization and its application to the design of diffraction grating filters," Opt. Lett. 32, 894-896 (Apr 2007).

[28] Shokooh-Saremi, M. and Magnusson, R., "Leaky-mode resonant reflectors with extreme bandwidths," Opt. Lett. 35, 1121-1123 (Apr 2010).

[29] Kennedy, J. and Eberhart, R., "Particle swarm optimization," in [Neural Networks, 1995. Proceedings., IEEE International Conference on], 4, $1942-1948$ vol.4 (nov/dec 1995).

[30] Chevallier, C., Fressengeas, N., Genty, F., and Jacquet, J., "Mid-infrared sub-wavelength grating mirror design: tolerance and influence of technological constraints," Journal of Optics 13(12), 125502 (2011).

[31] Elishakoff, I., Haftka, R., and Fang, J., "Structural design under bounded uncertainty-optimization with anti-optimization," Computers \& Structures 53(6), 1401 - 1405 (1994). 